\title{
Avoidance and contextual learning induced by a kairomone, a pheromone and a common odorant in female CD1 mice
}

\section{OPEN ACCESS}

Edited by:

Thomas Endres,

Otto-von-Guericke University,

Germany

Reviewed by:

Jeffrey B Rosen,

University of Delaware, USA

Pablo Chamero,

University of Saarland, Germany

*Correspondence:

Carmen Agustín-Pavón,

Unitat Pre-departamental de

Medicina, Universitat Jaume I, Av. de

Vicent Sos Baynat, s/n, 12071

Castelló de la Plana, Spain deagusti@uji.es

${ }^{\dagger}$ Present Address:

Lluís Fortes-Marco,

Departamento de Neurobiología

Molecular, Celular y del Desarrollo.

Instituto Cajal, Madrid, Spain

Specialty section:

This article was submitted to

Systems Biology,

a section of the journal

Frontiers in Neuroscience

Received: 25 June 2015

Accepted: 07 September 2015

Published: 06 October 2015

Citation:

Fortes-Marco L, Lanuza E,

Martínez-García F and

Agustín-Pavón C (2015) Avoidance and contextual learning induced by a

kairomone, a pheromone and a common odorant in female CD1 mice.

Front. Neurosci. 9:336.

doi: 10.3389/fnins.2015.00336

\author{
Lluís Fortes-Marco ${ }^{1,2+}$, Enrique Lanuza ${ }^{2}$, Fernando Martínez-Garcia ${ }^{1}$ and \\ Carmen Agustín-Pavón ${ }^{1 *}$ \\ ${ }^{1}$ Unitat Pre-departamental de Medicina, Facultad de Ciencias de la Salud, Universitat Jaume I, Castelló de la Plana, Spain \\ ${ }^{2}$ Departament de Biologia Cel.lular, Facultat de Ciències Biològiques, Universitat de València, València, Spain
}

Chemosignals mediate both intra- and inter-specific communication in most mammals. Pheromones elicit stereotyped reactions in conspecifics, whereas kairomones provoke a reaction in an allospecific animal. For instance, predator kairomones elicit anticipated defensive responses in preys. The aim of this work was to test the behavioral responses of female mice to two chemosignals: 2-heptanone (2-HP), a putative alarm pheromone, and 2,4,5-trimethylthiazoline (TMT), a fox-derived putative kairomone, widely used to investigate fear and anxiety in rodents. The banana-like odorant isoamyl acetate (IA), unlikely to act as a chemosignal, served as a control odorant. We first presented increasing amounts of these odorants in consecutive days, in a test box in which mice could explore or avoid them. Female mice avoided the highest amounts of all three compounds, with TMT and IA eliciting avoidance at lower amounts (3.8 pmol and $0.35 \mu \mathrm{mol}$, respectively) than $2-\mathrm{HP}(35 \mu \mathrm{mol})$. All three compounds induced minimal effects in global locomotion and immobility in this set up. Further, mice detected 3.5 pmol of TMT and IA in a habituation-dishabituation test, so avoidance of IA started well beyond the detection threshold. Finally, both TMT and IA, but not 2-HP, induced conditioned place avoidance and increased immobility in the neutral compartment during a contextual memory test. These data suggest that intense odors can induce contextual learning irrespective of their putative biological significance. Our results support that synthetic predator-related compounds (like TMT) or other intense odorants are useful to investigate the neurobiological basis of emotional behaviors in rodents. Since intense odorants unlikely to act as chemosignals can elicit similar behavioral reactions than chemosignals, we stress the importance of using behavioral measures in combination with other physiological (e.g., hormonal levels) or neural measures (e.g., immediate early gene expression) to establish the ethological significance of odorants.

Keywords: aversion, isoamyl acetate, 2,4,5-trimethylthiazoline, 2-heptanone, kairomones, pheromones, place conditioning, vomeronasal 


\section{Introduction}

Rodents are widely used in studies on the neurobiological basis of emotional behaviors. Chemical signals are the most relevant sensory cues for rodents, capable of eliciting strong emotional responses in them. For example, chemical signals such as alarm pheromones and predator kairomones are anxiogenic for mice and rats. Alarm pheromones are substances released by an injured or threatened animal and detected by conspecifics (Gutiérrez-García et al., 2007; Brechbühl et al., 2013), whereas predator kairomones elicit defensive responses in preys (for a review, see Fortes-Marco et al., 2013). Thus, experiments exposing mice and rats to alarm pheromones and kairomones are valuable to investigate the neural circuits controlling fear and anxiety, key features of pathologies such as generalized anxiety disorder, depression, or post-traumatic stress disorder.

The volatile chemical 2-heptanone (2-HP) has been proposed to act as an alarm substance in bees (Collins et al., 1989) and rats (Gutiérrez-García et al., 2007). This ketone is a component of the urine of rats, and its concentration is higher in stressed individuals. Urine from stressed rats or 2-HP alone induce stresslike reactions in recipient subjects (Gutiérrez-García et al., 2006), and despair in rats subjected to the forced swim paradigm (Gutiérrez-García et al., 2007). In mice, the concentration of 2HP in urine is dependent on the adrenal gland (Novotny et al., 1986), and it acts as a puberty modulator in females in a blend with other chemicals (Novotny et al., 1986; Jemiolo et al., 1989). Thus, 2-HP might be classified as a trigger pheromone for rats and a primer pheromone for mice, following the definition by McClintock (2002). To our knowledge, the behavioral responses of mice to 2-HP have not been characterized yet, and most of the studies using 2-HP have focused on the mechanism of detection (Boschat et al., 2002; Thompson et al., 2004). Interestingly, a recent paper suggests that, in mice, some substances proposed as alarm pheromones, like 2-sec-butyl-4,5-dihydrothiazole, share structural similarity with kairomones (but see Jemiolo et al., 1985 on the possible role of this chemical as a sexual pheromone). Thus, alarm pheromones and kairomones might act using the same neural circuits conveying signals of danger (Brechbühl et al., 2013).

Cat fur and feces are potential sources of kairomones for rodents. Indeed, cat odors consistently induce biochemical and behavioral measures of stress in mice and rats, such as elevation of plasma glucocorticoids, fear responses such as freezing, avoidance, and contextual memory (Berton et al., 1998; MuñozAbellán et al., 2008, 2009). However, the components of cat fur odor and feces are diverse, not yet fully characterized, and the reaction to cat odor depends on the donor cat (MuñozAbellán et al., 2008) and its diet (Berton et al., 1998; Ferrero et al., 2011). Conversely, specific odorants derived from predator sources would offer the advantage of a controllable presentation to produce reproducible results.

The synthetic molecule 2,5-dihydro-2,4,5-trimethylthiazoline (TMT), a component isolated from fox feces (Vernet-Maury, 1980), offers the mentioned advantages. TMT induces freezing, contextual learning, and anxiety-related behavioral changes in rodents (Endres and Fendt, 2007, 2009). Moreover, TMT activates brain regions related to stress and anxiety, although the activation pattern differs from that induced by cat odor (Dielenberg et al., 2001; Staples et al., 2008; Janitzky et al., 2015; Pérez-Gómez et al., 2015). This fact, together with the fact that both unconditioned responses to TMT and its ability to support conditioning are dependent on the environment and protocol used (Wallace and Rosen, 2000; Morrow et al., 2002; Blanchard et al., 2003; Endres and Fendt, 2007), have led to some debate on the kairomonal or simply aversive nature of this molecule (Fendt and Endres, 2008; Fortes-Marco et al., 2013).

In this vein, we have previously hypothesized that the exposure to intense odorants might be indeed very aversive, so it could elicit similar anxiety-like responses to the ones elicited by kairomones and alarm pheromones in mice-not necessarily accompanied by behavioral components of fear i.e., freezing (Fortes-Marco et al., 2013).

Thus, we sought to compare the behavioral reactions of female mice to increasing amounts of TMT, 2-HP and isoamyl acetate (IA), a banana-like odorant frequently used as a control odor (Wallace and Rosen, 2000; Root et al., 2014) in twochoice tests. Previous studies from our lab have validated these tests to investigate the attractive properties of male pheromones for female mice (Agustín-Pavón et al., 2007, 2014; Martínez-Ricós et al., 2007). We hypothesized that the putative kairomone TMT would be avoided at detectable concentrations. The putative pheromone 2-HP might be either attractive or aversive depending on its role-alarm pheromone or puberty modulator, see above. Finally, the control odorant should not elicit avoidance, except perhaps at high concentrations. We also hypothesized that sufficiently aversive olfactory stimulation might be able to support learning in the animals. Thus, we tested whether repeated exposure to the concentrations eliciting the maximum behavioral reaction could induce a conditioned contextual avoidance in mice.

\section{Materials and Methods}

\section{Animals}

For the present experiments, we used 125 adult female CD1 mice (8-18 weeks old, Janvier SAS, St Berthevin Cedex, France). Animals were housed under controlled temperature $\left(22-24^{\circ} \mathrm{C}\right.$ ) and a light-dark cycle 12:12 (light from 08:00 to 20:00), with food and water ad libitum. Animals were treated throughout according to the European Communities Council Directive of (86/609/EEC), and the protocols were approved by the Committee of Ethics on Animal Experimentation of the University of Valencia.

\section{Odorants}

We used three odorants: 2,5-dihydro-2,4,5-trimethylthiazoline (TMT; Contech, Victoria, Canada), a putative kairomone found in fox feces and detected by the main olfactory system and the Grueneberg ganglion (Brechbühl et al., 2013), 2-heptanone (2HP, Sigma-Aldrich, Schnelldorf, Germany), a putative mouse pheromone detected by the main and accessory olfactory epithelia (Thompson et al., 2004; Xu et al., 2005), and isoamyl acetate (IA, Panreac Quimica SA, Barcelona, Spain), a control 
odorant detected by the main olfactory system (Xu et al., 2005) and unlikely to participate in intra or interspecific communication (Root et al., 2014). We diluted the odorants to the desired concentration, with PBS with Triton X-100 1\%, $(\mathrm{pH}=$ 7.4) for 2-HP and TMT and mineral oil for IA. We selected these solvents because the odorants were more readily diluted in them than in distilled water. In addition, mice were not able to detect differences between the odorants diluted in the solvents or in distilled water (Supplementary Material, Experiment S1). Also, the solvents did not elicit enhanced chemoinvestigation (see Section Results and Figure 2).

\section{Behavioral Tests \\ Experiment 1. Behavioral Effects of Increasing Amount of Odorants}

To check the behavioral reaction of mice toward each amount of odorant in two-choice tests, subjects were randomly assigned to four groups. Each group was presented with one of the odorants or PBS (control) (TMT $n=12,2$-HP $n=11$, IA $n=12$, PBS $n=12$ ). The behavioral test was performed in a methacrylate opaque box $(45 \times 47.5 \times 22.5 \mathrm{~cm})$, divided in two identical chambers by a panel with a door to minimize diffusion of the odors. Each chamber had one perforated stainless steel capsule (JP Selecta, Abrera, Barcelona, Spain) attached to the floor with double-sided adhesive tape. We prepared serial dilutions of each of the odorants, from the pure substance to $10^{-8}$, and pipetted $5 \mu \mathrm{l}$ of the corresponding solution in a piece of filter paper $(2 \times$ $2 \mathrm{~cm}$ ) inside each capsule. The capsule on the stimulus chamber contained $5 \mu$ l of the corresponding odorant, whereas the capsule on the neutral chamber contained the corresponding solvent, except in the PBS group, in which both capsules contained $5 \mu \mathrm{l}$ of PBS.

Animals were habituated to the experimenter and apparatus for $10 \mathrm{~min}$ for 3 days. The fourth day, we performed a 5 -min test (control), in which both capsules contained the correspondent solvent (PBS with Triton X-100 or mineral oil, see above) or PBS. On the following days, mice were exposed to the $5 \mu \mathrm{L}$ of the relevant chemical stimulus at increasing concentrations at the stimulus chamber (exposure days 1-9), and $5 \mu \mathrm{L}$ of the corresponding solvent in the capsule at the neutral chamber. All odorants have similar molecular weight and density (TMT 129.2, $1 \mathrm{~g} / \mathrm{ml}$; 2-HP 114.2, $0.8 \mathrm{~g} / \mathrm{ml}$; IA $130.2,0.9 \mathrm{~g} / \mathrm{ml}$ ), so the molar concentration of the pure substance is similar (TMT 7.7 M, 2-HP and IA, $7 \mathrm{M}$ ). Thus, the first exposure day, mice had access to 0.38 pmol of TMT or 0.35 pmol of 2-HP and IA, and the amount of odorant was increased 10 -fold each day to the pure substance, i.e., $38 \mu \mathrm{mol}$ of TMT or $35 \mu \mathrm{mol}$ of $2-\mathrm{HP}$ and IA. To avoid diffusion of the odorant and facilitate exhausting the volatiles of the room, we performed the tests in a room with mild negative air pressure. Experimental cages were thoroughly cleaned after each test, and each stimulus was used in a different room.

\section{Experiment 2. Habituation-Dishabituation Tests}

To establish the detection threshold of each of the odorants, we submitted three separate groups of mice $(n=6$ for each odorant) to habituation-dishabituation tests, following AgustínPavón et al. (2007).
The tests were performed in a squared opaque methacrylate box $\left(25 \mathrm{~cm}^{2}\right)$ with a hole at $8 \mathrm{~cm}$ from the floor in one of the walls. Females were placed in the test box $3 \mathrm{~min}$ before the test for habituation. The stimuli were presented to the mice in a stick with a cotton swab at the tip, which was introduced through the hole and fixed to the box wall. We presented the mice with three consecutive 1-min presentations of the swab impregnated with $5 \mu \mathrm{l}$ of water, followed by three consecutive presentations of $5 \mu \mathrm{l}$ each odorant amount. Between each odorant amount, mice were presented with three consecutive 1-min presentations of the corresponding solvent. Mice investigate more each stick when presented for the first time or when its odor changes, and investigation decreases in successive presentations. Thus, this is a reliable method to investigate the detection threshold of odorants.

\section{Experiment 3. Place Conditioning Test}

To test whether repeated exposure to the odorants in the same location would induce learning, we performed a place conditioning test following the protocol described in MartínezRicós et al. (2007). Animals were randomly distributed in three groups, (TMT, $n=11$; 2 -HP, $n=12$, IA, $n=12$ ). We used the same test box as in Experiment 1, so that animals could freely explore both chambers. Animals were habituated to the experimental conditions for $10 \mathrm{~min}$ for 2 days. The third day, we put $5 \mu \mathrm{L}$ PBS in each capsule and recorded the behavior of the animals for $5 \mathrm{~min}$ (control). From the next day, mice had access for four consecutive days (training days, 1-4) to $5 \mu \mathrm{L}$ of their corresponding odorant at pure concentration in the stimulus chamber and to PBS in the neutral chamber, for $5 \mathrm{~min}$. The day after the last training session, we evaluated the induction of contextual memory (test) with PBS in each capsule.

\section{Behavioral Measures}

All tests were video-recorded and the videos were analyzed with the video-tracking software SMART v2.5.11 (Panlab, Cornella, Spain). For experiments 1 and 3, we defined an area of interest covering $25 \%$ of each chamber surface, as a circular region of $12 \mathrm{~cm}$ of radius surrounding the center of the capsule (stimulus or neutral zones). This zone ensured the detection of the animal in close proximity of the stimuli.

For each stimulus and test, we obtained data from time spent in the area of interest and distance traveled in $\mathrm{cm}$. Informal observations by a trained observer who was blind to the experimental conditions revealed lack of risk assessment postures or freezing, so we used the percentage of time that the animal moved at a speed $<1 \mathrm{~mm} / \mathrm{s}$ as an approximate measure of immobility (Fortes-Marco et al., 2013). As a measure of attraction/avoidance, we calculated an avoidance ratio as the ratio between the time spent on the stimulus zone and the total time spent in the neutral plus the stimulus zone (see Agustín-Pavón et al., 2014). A value of 0.5 of this avoidance ratio indicates that the stimulus is neither attractive nor avoided, whereas an avoidance ratio $<0.5$ reveals avoidance of the stimuli. For experiment 2, the tests were videotaped and an observer blind to the experimental conditions measured the time that females 
spent rearing on their hind limbs and actively sniffing at the cotton tip.

\section{Statistical Analysis}

Data were analyzed with R statistical software (v. 3.1.2, http:// www.R-project.org/) and IBM SPSS 22.0. We checked the normality and homocedasticity of the data by means of a Kolmogorov-Smirnov and Levene test. Data from Experiment 1 were analyzed by means of ANOVA for repeated measures, with the factors DAY/CONCENTRATION (for the avoidance ratio) and ZONE (for time spent in the zones) as withinsubject factors, followed by Dunnet post-hoc comparisons (to compare exposure days with the control condition) or posthoc pairwise comparisons with the Bonferroni correction. Data from Experiment 2 were analyzed by means of paired Student's $t$-tests between the last presentation of each solvent and the first presentation of the following odorant. Data from Experiment 3 were analyzed by means of paired Student's $t$-test (avoidance ratio in control vs. place avoidance test), repeated measures ANOVA (distance traveled and immobility) with DAY and ZONE as within-subjects factors, and repeated measures ANOVA (avoidance ratio during exposure days) with DAY as within-subject factor and ODOR as between-subjects factor.

\section{Results}

\section{Experiment 1. Behavioral Effects of Increasing Amount of Odorants}

The aim of the first experiment was to determine the range of amounts at which each odor would elicit a measurable behavioral reaction in mice, including attraction/avoidance, distance traveled, and immobility, in two-choice tests. To ensure that there was no a priori preference of the animals for any compartment-this was unlikely, since both were identical-, or some habituation process that would affect the activity of the animals across tests, we run group of animals that were exposed to PBS in each zone for 10 consecutive days. None of the behavioral measures varied across days in these mice exposed to PBS alone, so any behavioral changes in the mice exposed to the different odorants could be attributed to the effects of the stimuli. (Supplementary Material, Experiment S2).

The avoidance ratio for the group exposed to TMT was significantly different from control at the highest amount of TMT used [repeated measures ANOVA, factor CONCENTRATION, $F_{(9,99)}=4.9, p<0.001 ;$ post-hoc comparison between exposure day $9(38 \mu \mathrm{mol}$ of TMT) vs. control (no TMT), $p=$ 0.009; Figure 1A]. We further checked whether there was a difference in the raw time that animals spent in each zone. There was a significant decrease of the time spent in the stimulus zone when it contained $38 \mu \mathrm{mol}$ of TMT with respect to the stimulus zone in the control day [repeated measures ANOVA, CONCENTRATION $\times$ ZONE, $F_{(9,99)}=3.6, p<0.001$; post-hoc comparison for exposure day 9 , stimulus zone vs. control, $p=$ 0.001; Figure 1B]. Still, post-hoc pairwise comparisons between zones showed that time spent in the TMT zone was lower with respect to the neutral zone during exposure days $2,4,8$, and 9 (3.8 pmol, $p=0.023$; $0.38 \mathrm{nmol}, p=0.015 ; 3.8 \mu \mathrm{mol}, p=0.002$; and $38 \mu \mathrm{mol}, p<0.001$; Figure 1B). These results suggest that mice were able to detect TMT from $3.8 \mathrm{pmol}$, since this amount induced a slight avoidance reaction (Figure 1B), but TMT was strongly avoided at pure concentration only as measured with both time spent in zones and avoidance ratio.

The putative pheromone 2-HP induced avoidance at the highest amount presented [repeated measures ANOVA of the avoidance ratio, CONCENTRATION, $F_{(9,90)}=9.4, p<0.001$, post-hoc comparison exposure day 9 vs. control, $p=0.001$; Figure 1C]. Further, the ANOVA of the raw time spent in zones and subsequent post-hoc tests revealed an increase in time spent in the neutral zone and a decrease in the stimulus zone with $35 \mu \mathrm{mol}$ of 2 -HP [CONCENTRATION $\times \mathrm{ZONE}, F_{(9,90)}=7.4$, $p<0.001$, post-hoc exposure day 9 vs. control, neutral zone $p=0.009$, stimulus zone $p=0.03$; Figure 1D]. Pairwise post-hoc comparisons between zones confirmed a significant difference of time spent in stimulus vs. neutral zone in exposure day 9 only $(p<0.001)$.

Finally, mice also avoided IA at the highest amount presented [repeated measures ANOVA for the avoidance ratio, CONCENTRATION, $F_{(9,90)}=9.1, p<0.001$; post-hoc comparison of exposure day 9 vs. control, $p=0.048$; Figure 1E]. For the time spent in each zone, there was a decrease in time spent in the stimulus zone in the presence of $35 \mu \mathrm{mol}$ of IA with respect to the control [ANOVA, CONCENTRATION $\times$ ZONE, $F_{(9,90)}=5.83, p<0.001$, post-hoc comparison of exposure day 9 vs. control, $p=0.002]$. In addition, post-hoc pairwise comparisons revealed significantly lower time spent in the stimulus zone as compared to the neutral zone when IA was presented from 0.35 to $35 \mu \mathrm{mol}(p=0.014 ; p=0.004 ; p<$ 0.001, respectively; Figure 1F). Thus, the pattern of avoidance of IA resembles that of TMT.

Surprisingly, an ANOVA between tests revealed that TMT did not significantly affect distance traveled $\left[F_{(9,90)}=1.7, p=\right.$ $0.11]$ or percentage of immobility $\left[F_{(9,90)}=1.5, p=0.22\right]$. By contrast, distance traveled was, overall, significantly decreased by the increasing concentrations of 2-HP [DAY effect, $F_{(9,90)}=4.0$, $p<0.001]$. Post-hoc comparisons revealed significant decreases in the presence of $0.35 \mathrm{nmol}$ to $3.5 \mu \mathrm{mol}$ of $2-\mathrm{HP}$ with respect to the control (all $p<0.05$ ). The percentage of immobility was also significantly different across tests $\left[\mathrm{DAY}, F_{(9,90)}=2.2\right.$, $p=0.027$. Thus, 2 -HP was avoided by mice at the highest presented amount, but concentration-dependently affected the activity of the animals. Finally, the exposure to IA significantly decreased the distance traveled and increased immobility globally across tests $\left[F_{(9,90)}=3.4, p=0.018, F_{(9,90)}=3.3, p=0.026\right.$; Table 1].

\section{Experiment 2. Habituation-dishabituation test}

Results from Experiment 1 suggested that mice could detect 3.8 pmol of TMT, since this was the minimal amount that elicited a slight avoidance response. However, both 2-HP and IA were not avoided until we presented 35 and $0.35 \mu \mathrm{mol}$, respectively. To investigate whether odorants affected the behavior of mice at their olfactory detection threshold or beyond, we carried 


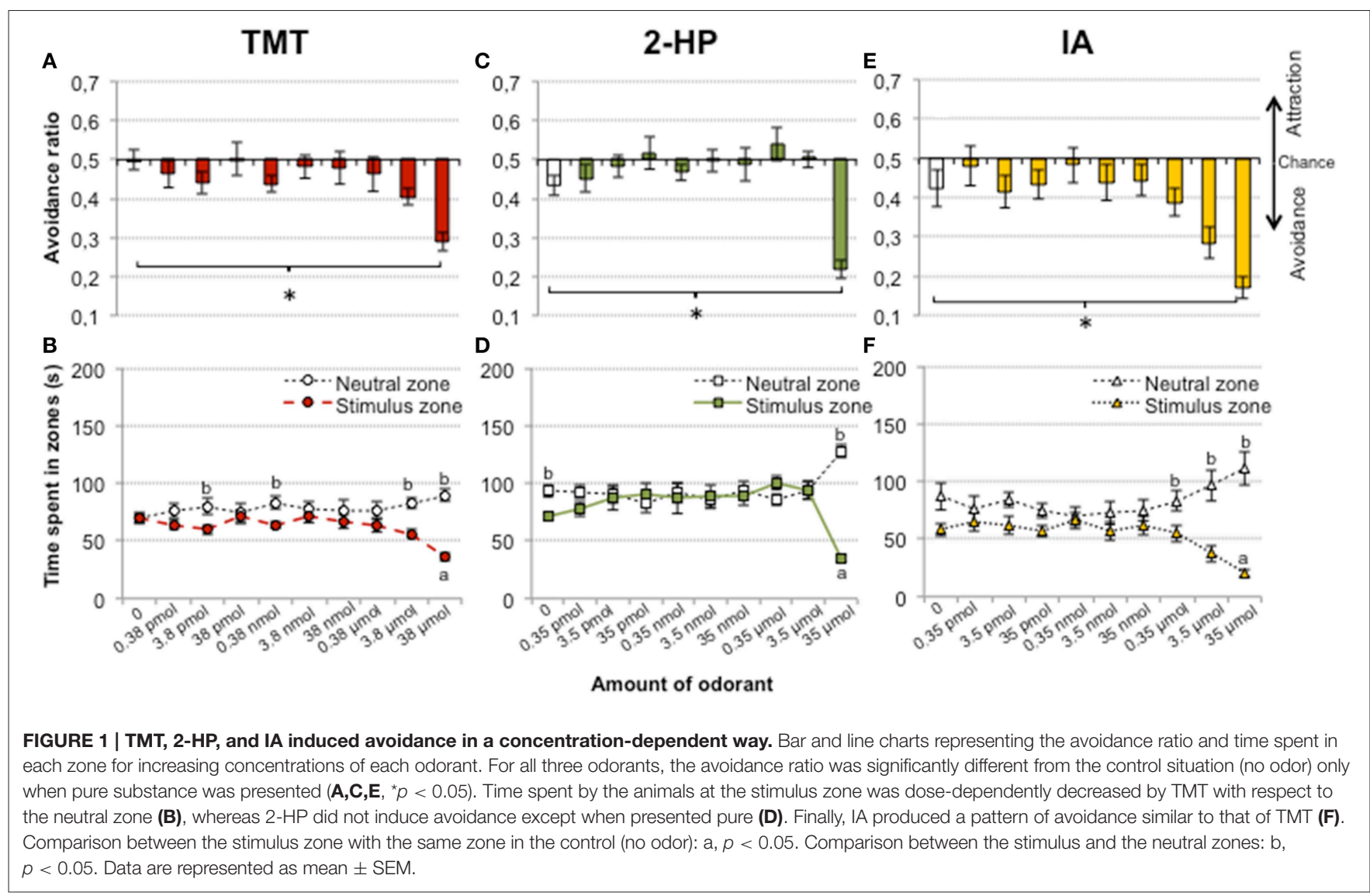

out a habituation-dishabituation test. The analysis comparing the last presentation of the cotton swab impregnated with solvent with the first presentation of each amount of odorant tested revealed that female mice detected 3.8 pmol of TMT, since this amount increased investigation of the cotton swab ( $p=0.002$; Figure 2A). By contrast, 2-HP did not significantly increased investigation until $35 \mu \mathrm{mol}$ were presented $(p=$ 0.039; Figure 2B). Finally, $3.5 \mathrm{pmol}$ of IA significantly increased chemoinvestigation $(p=0.005)$. Thus, we confirmed that mice detected 3.8 pmol of TMT, although avoidance behavior was not strong with this amount of odorant. Further, in agreement with the avoidance expressed by mice in Experiment 1, 2HP did not significantly enhanced chemoinvestigation until presented at the maximum amount (see also Supplementary Material). By contrast, IA was detected in the habituationdishabituation test much more diluted than it was avoided in Experiment 1 . These results also raise the possibility that learning could contribute to the strong avoidance displayed toward TMT and IA at the highest amount used. Finally, this experiment confirmed that the solvents did not elicit chemoinvestigation (Figure 2).

\section{Experiment 3. Place Conditioning Test}

We next checked whether the repeated exposure to the different stimuli was able to induce the formation of a contextual memory. To do so, we used the undiluted odorants, which were equally avoided by mice, in a place conditioning experiment.
Mice of the TMT group expressed a conditioned avoidance for the stimulus chamber after four consecutive days of exposure to this kairomone (Student's $t$-test of the avoidance ratio control vs. place conditioning test, $p=0.029$; Figure 3A). Moreover, a Student's $t$-test against the chance value 0.5 revealed that the avoidance ratio was significantly different from chance in the test ( $p=0.027$ ) but not the pre-training control $(p=0.64)$. However, neither distance traveled nor global percentage of immobility were significantly different between the control and the place conditioning test ( $p=0.7$, Figure 3B). We further explored whether the percentage of immobility would be dependent on the zone, i.e., whether mice would stay inactive in the neutral or the stimulus zone (Fortes-Marco et al., 2013). Indeed, there was a significant difference between zones in the place conditioning test, driven by an increase in the percentage of immobility in the neutral zone in the test with respect to control [repeated measures ANOVA, DAY $\times$ ZONE, $F_{(1,10)}=8.7, p=0.014$, post-hoc stimulus vs. neutral zone in the test, $p=0.002$; post-hoc time spent in neutral zone in control vs. test, $p=0.017$; Figure $3 \mathrm{C}$ ].

In spite of being strongly avoided at the highest amount presented, 2-HP did not induce contextual memory (avoidance ratio, control vs. test $p=0.99$; Figure 3D). Distance traveled and immobility were not significantly affected either $(p=0.068$ and 0.094, respectively; Figures 3E,F).

Finally, in the group exposed to IA the decrease of the avoidance ratio did not reach statistical significance with respect to the control ( $p=0.067$; Figure 3G). However, a Student's 
$t$-test against the chance value revealed that the avoidance ratio was significantly different from chance at the test $(p=0.001)$ but not the control $(p=0.115)$, so this measure indicates the formation of a conditioned avoidance. Further, distance traveled was significantly lower in the test with respect to the control $(p=$ 0.034; Figure $3 \mathrm{H}$ ). The percentage of immobility in each zone was different in the memory test, due to an increase in immobility in the neutral zone $\left[\mathrm{DAY} \times \mathrm{ZONE}, F_{(1,11)}=4.9 p=0.049\right.$, stimulus vs. neutral chamber, $p=0.004$; time in immobility in the neutral zone control vs. test, $p=0.012$; Figure 3I).

In summary, TMT and IA induced a contextual memory after repeated exposure, so that mice avoided the stimulus zone even in the absence of the odorants. Exposure to IA significantly decreased distance traveled in the contextual memory test, and both TMT and IA increased the immobility of the animals in the neutral zone, paralleling the significant avoidance of the stimulus zone. Conversely, exposure to 2-HP did not produce statistically significant behavioral differences between the control and the memory test.

To check whether the different effects of each odorant in memory and activity were due to differential effects of the repeated exposure to them, we compared the avoidance ratio, distance traveled and immobility during the training days between groups (TMT, 2-HP and IA). The avoidance ratio of the groups exposed to TMT and 2-HP was significantly different to those of IA [repeated measures ANOVA, DAY $\times$ ODOR, $F_{(6,96)}=3.1, p=0.01$; post-hoc comparison TMT vs. IA and 2-HP and 2-HP vs. IA, both $p=0.023$; Figure 4A]. Further post-hoc pairwise comparisons between the individual exposure days revealed that all odors were equally avoided during the first two training days, but from day 3 , the avoidance ratio of TMT decreased and became significantly different from both 2-HP and IA $(p<0.05$ in all cases; Figure 4A). In fact, the avoidance ratio in the TMT group was significantly different between day 2 and $3(p=0.042)$. Conversely, the avoidance ratio of the group exposed to 2-HP was significantly lower in day 2 than in day 1 $(p=0.02)$. Finally, the avoidance ratio of the group exposed to IA did not vary across days. These findings suggest that mice were slightly habituated to the aversive properties of TMT, whereas they expressed a higher avoidance of 2-HP in consecutive tests.

Distance traveled was significantly different across exposure days and between groups [repeated measures ANOVA, ODOR, $F_{(2,32)}=5.4, p=0.009 ;$ TEST $\times$ ODOR, $F_{(6,96)}=3.1$, $p=0.01$; Figure 4B]. Post-hoc comparisons of the factor ODOR showed that, overall, the distance traveled by the mice exposed to TMT was significantly different to distance traveled by the mice exposed to 2-HP $(p=0.007)$. Further post-hoc pairwise comparisons revealed that distance traveled by animals exposed to 2-HP was lower at day 4 as compared to day $1(p=0.014)$, whereas mice exposed to IA traveled less distance all three last exposure days as compared to the first one (all $p<0.05$ ). We thus checked whether these differences were due to basal differences in distance traveled between the groups. Importantly, distance traveled was similar for all groups at the control day $(p>0.1)$. Further, the ANOVA followed by Dunnet post-hoc comparison revealed that TMT increased distance traveled during the first exposure with respect to the control $(p=0.019)$, whereas $2-\mathrm{HP}$ 


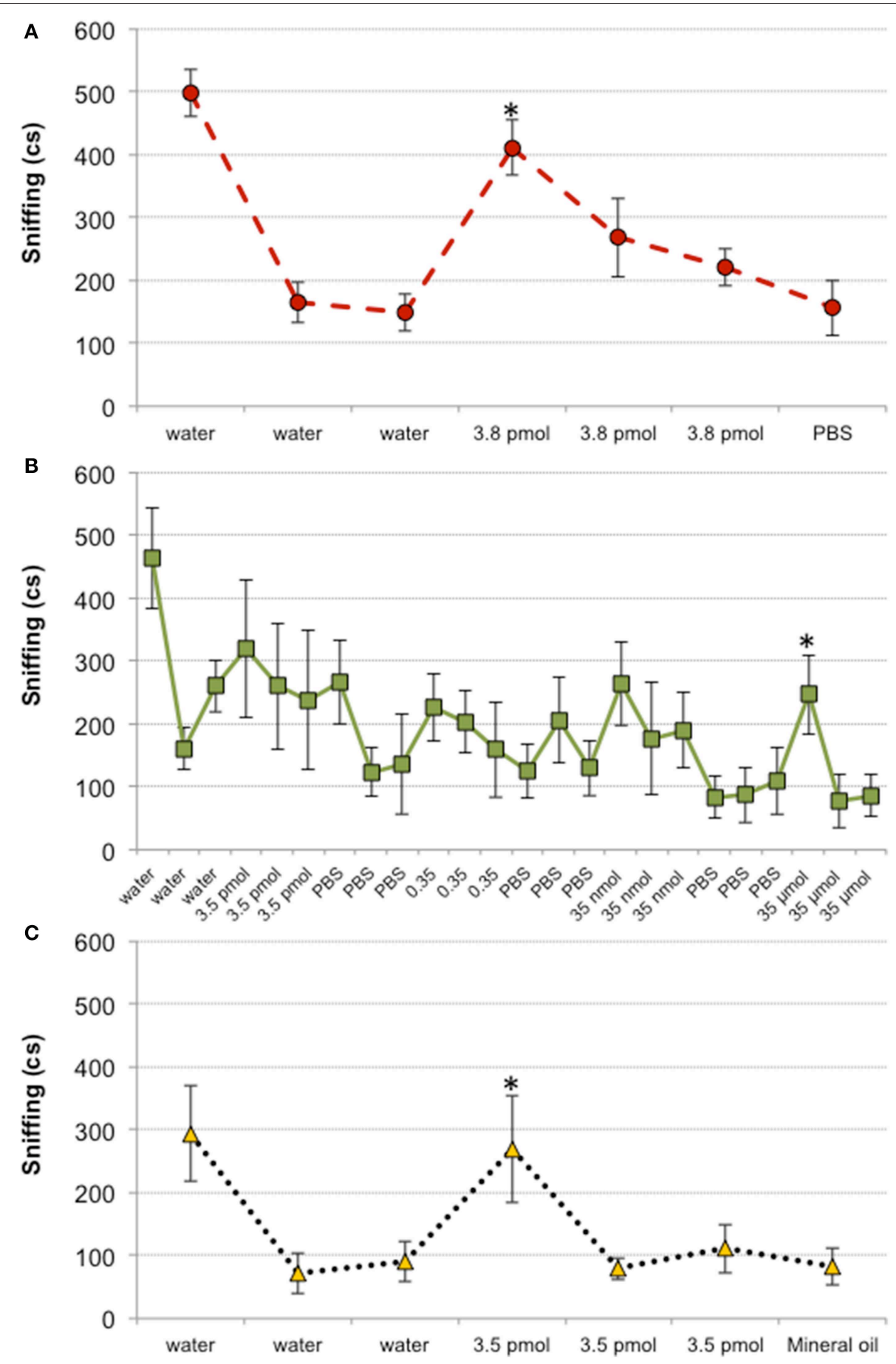

FIGURE 2 | Mice are able to detect TMT, 2-HP and IA in habituation-dishabituation tests. Line charts representing the chemoinvestigation of scented cotton swabs in cs. 3.8 pmol of TMT elicited increased chemoinvestigation (A), whereas 2-HP did not significantly increase chemoinvestigation until we presented $35 \mu$ mol (B). The detection threshold of IA was similar to that of TMT (C). Comparison between last presentation of a solvent (water, PBS with Triton X-100 $1 \%$ or mineral oil) and first presentation of each odorant amount, ${ }^{*} p<0.05$. Data are expressed as mean $\pm \mathrm{SEM}$.

and IA decreased distance traveled with respect to the control at days 3 and 4 , respectively (both $p=0.04$ ).

As expected, immobility followed a pattern that was complementary to that of distance traveled [repeated measures
ANOVA, TEST $\times$ ODOR, $\left.F_{(6,96)}=2.3, p=0.042\right]$. Overall, immobility was significantly different in the TMT and 2-HP groups $(p=0.037$ ). Again, these differences were not due to different initial levels of immobility, since this measure was not 

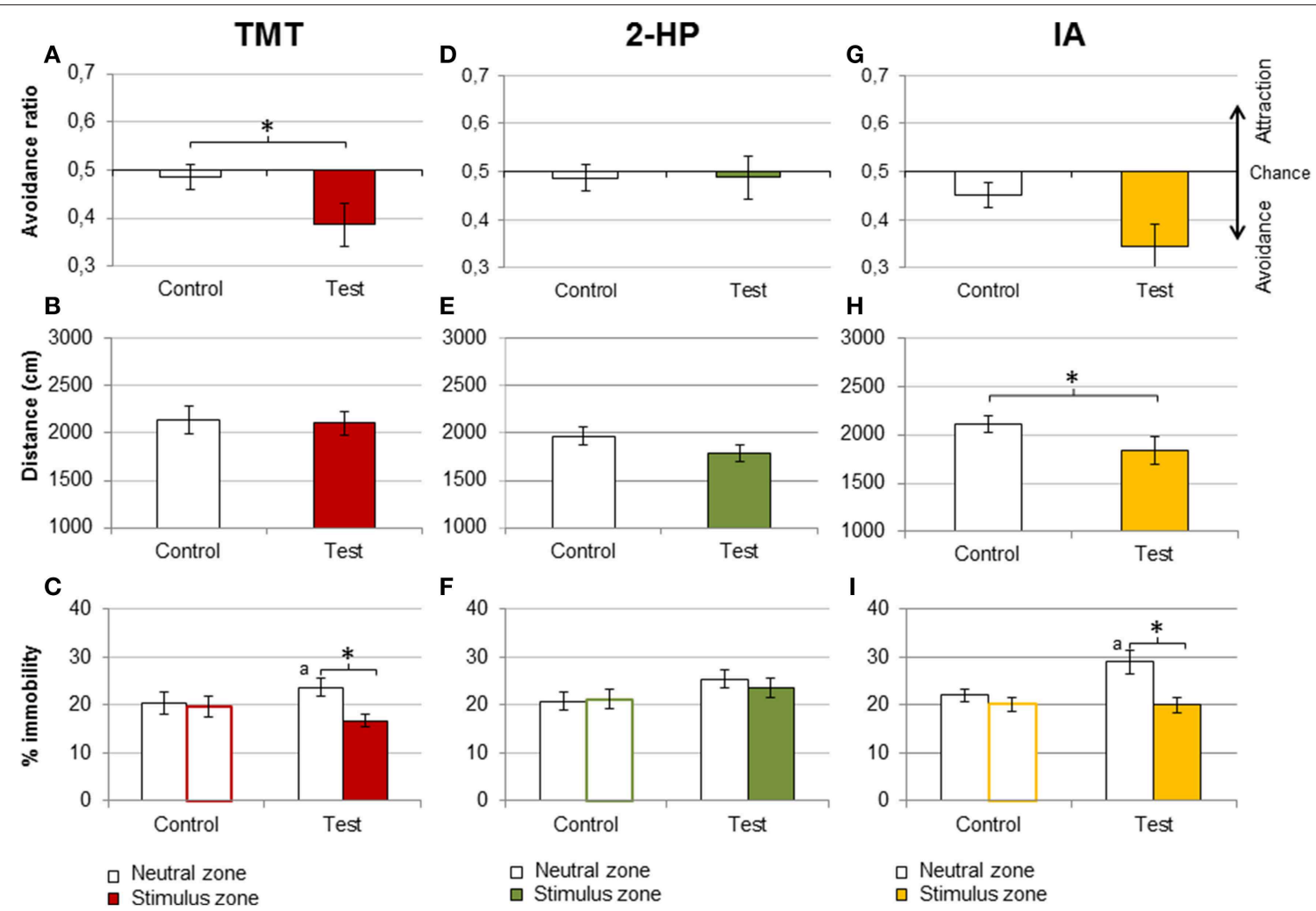

FIGURE 3 | TMT and IA, but not 2-HP, induced contextual learning. Bar charts representing behavioral responses in the contextual learning experiment, in the pre-exposure control (Control) and the contextual memory test (Test). Mice exposed to pure TMT during four consecutive sessions expressed conditioned place avoidance to the zone paired with the stimulus, as shown by a significant decrease of the avoidance ratio in the test (A). In addition, TMT failed to affect distance traveled (B), but increased immobility in the neutral zone during the test (C), suggesting that animals avoided TMT and stayed immobile in the neutral zone. By contrast, 2-HP did not induce a contextual memory, since it did not affect the avoidance ratio (D), but, overall, it slightly decreased distance traveled (E), and did not affect immobility (F). Finally, IA produced a marginally significant decrease in the avoidance ratio (G), and significantly decreased distance traveled (H). Like TMT, IA increased immobility in the neutral zone (I). ${ }^{*} p<0.05$. Comparison between the neutral zones in control and test: a, $p<0.05$. Data are expressed as mean \pm SEM.

significantly different between groups in the control day (all $p>0.9)$. In contrast to most reports showing an increase in freezing in animals exposed to TMT, this putative kairomone decreased immobility during the first day as compared to control $(p=0.017)$. Conversely, $2-\mathrm{HP}$ increased immobility at day 4 as compared to control $(p=0.004)$, whereas animals exposed to IA did not show any significant variation in the percentage of time they spent inactive (Figure 4C).

\section{Discussion}

Our results indicate that female mice displayed specific behavioral changes when exposed to substances thought to be implicated in inter (TMT) and intraspecific communication (2-HP), but also to a common odorant unlikely to act as a chemosignal (IA). Mice avoided these chemicals in a concentration-dependent way, but showed opposite changes in activity in response to them. Further, avoidance of both TMT and IA was expressed beyond the detection threshold of the odorants. In addition, only TMT and IA induced conditioned, context-dependent behavioral changes. We discuss these results by comparing them with the behavioral responses of mice to the common odorant IA.

\section{Exposure to TMT Elicits Avoidance but not Freezing}

Mice mainly rely on their sense of smell to avoid danger. Thus, when given the opportunity, they readily avoid spots containing predator kairomones (Root et al., 2014; Wernecke et al., 2015). Our results in female CD1 mice, showing that they avoid a zone containing TMT, a putative kairomone, are in agreement with this view. In fact, in Experiment 1, mice spent less time in the zone of the cage containing TMT than in the opposite neutral zone when TMT was present at amounts as low as $3.8 \mathrm{pmol}$ and $0.38 \mathrm{nmol}$ (Figure 1A). However, in the ensuing sessions at higher concentrations $(3.8 \mathrm{nmol}$ to $0.38 \mu \mathrm{mol})$ mice seemed to habituate and spent the same amount of time in both zones. Avoidance reappeared at the highest concentrations (3.8 and $0.38 \mu \mathrm{mol}$ ). In Experiment 2, we confirmed the olfactory detection of 3.8 pmol TMT, so that mice can detect and avoid TMT at low concentrations, supporting its role as a kairomone.

However, the time spent in the stimulus zone was significantly reduced with respect to the control situation (no odor) only when $38 \mu \mathrm{mol}$ TMT were presented (Figure 1B). In addition, mice displayed a similar avoidance pattern to TMT and to IA, an odorant unlikely to have a kairomonal role. In fact, IA is frequently used as a control odor for TMT studies (Wallace 


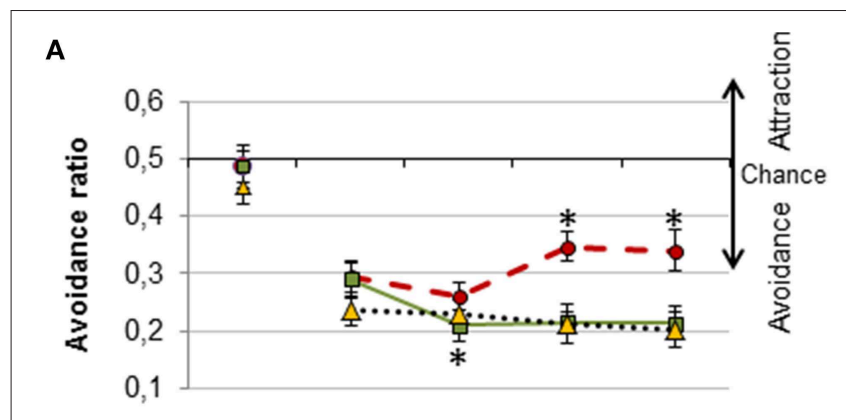

B

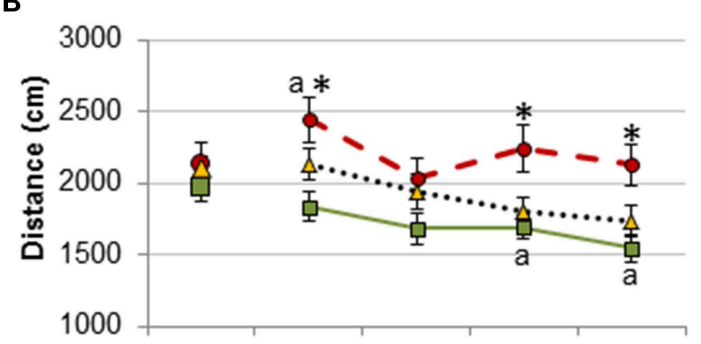

C

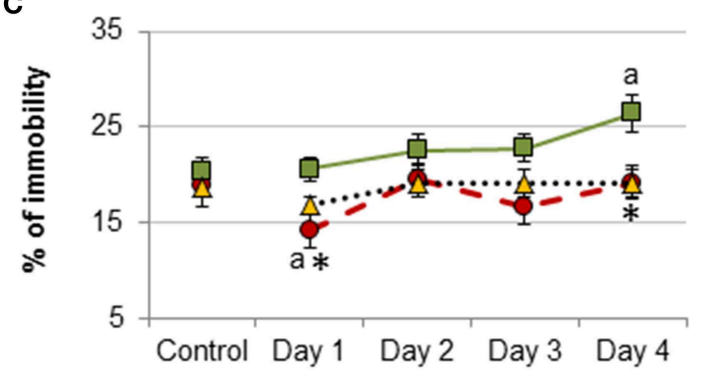

- TMT $\quad$ T2-HP $\quad \Delta \mathrm{IA}$

FIGURE 4 | Pure TMT, 2-HP and IA were avoided and differently affected locomotion. Line charts representing behavioral responses during the training days of the contextual conditioning experiment for each odorant. All three odors were significantly avoided, but the avoidance of TMT was blunted across sessions, whereas avoidance of 2-HP increased in the second day (A). Distance traveled was differentially affected by the three odors: whereas TMT increased locomotion during the first exposure day and then this parameter remained stable, 2-HP and IA decreased locomotion across tests (B). TMT reduced immobility the first training day, and 2-HP increased it during the last training day, whereas IA did not affect to this measure (C). Comparison between odorants: ${ }^{*} p<0.05$. Comparison between exposure days and the control situation: $a, p<0.05$. Data are expressed as mean \pm SEM.

and Rosen, 2000; Root et al., 2014), and our results showing that low amounts of IA are detected but not avoided by mice support its use as such, albeit only at those low concentrations. Our findings suggest that the behavioral reactions that mice display to supra-threshold, very intense odorants might not be odorant-specific, and therefore they may not be informative about the role of that substance as a chemical signal. In this line, a recent study by Dewan et al. (2013), showed that genetic deletion of specific olfactory receptors blocked the aversion that mice expressed for low, but not high, concentration of amines found in the urine of predators and for the urine itself. The high concentration of amines used in that study were pungent to humans, so the aversion could be due to either overactivation of non-specific olfactory receptors or trigeminal stimulation (see below), supporting that that aversion toward very intense odors might not only depend on the biological significance of the odorant. Second, this study highlights that biologically significant chemosignals are detected at very low concentrations.

Further, behavioral avoidance of a given stimulus does not necessarily reflect fear, but rather that the stimulus has aversive properties for the subject due to its repugnant, pungent or disgusting properties. In this sense, Endres and Fendt (2009) demonstrated that whereas both TMT and butyric acid elicited avoidance in rats, only TMT elicited freezing, a more accurate behavioral measure of fear.

However, TMT did not produce any freezing in our mice as measured by a trained observer. Immobility, a behavioral measure that could approximate freezing, was not affected either by the increasing concentrations of TMT. Quite the opposite, animals repeatedly exposed to pure TMT displayed heightened locomotion and reduced immobility, as compared to both the control situation (no odor) and to animals exposed to 2-HP. These findings contrast with the above mentioned study by Endres and Fendt (2009) and several other reports showing that rats (Wallace and Rosen, 2000) and mice (Hebb et al., 2004; Hacquemand et al., 2013) display enhanced immobility and freezing in the presence of TMT, but are in agreement with our previous findings in two strains of mice (Fortes-Marco et al., 2013). In fact, sensitivity to TMT is dependent on the strain in rats, so that, under the same conditions, Sprague-Dawley, but not Wistar rats display freezing in the presence of TMT (Rosen et al., 2006). In agreement, we have previously shown that mice of the C57BL/J6 strain displayed enhanced immobility in the presence of the TMT as compared to CD1 (Fortes-Marco et al., 2013).

The contrasting findings about freezing to TMT found in the literature also reflect that responses of animals toward a given aversive stimulus are critically dependent on the protocol used. To investigate this issue, Morrow et al. (2002) exposed rats to TMT either in a low anxiety (a comfortable, dimly lit open field) or a high anxiety environment (a bigger apparatus intensely lit). Their results showed that rats displayed enhanced immobility in the high anxiety environment only. Thus, the behavioral strategy that animals select to cope with an aversive stimulus is dependent on the possibilities offered by the environment. In a singlechamber open field or in the home cage, where the animals cannot escape or hide, it is more likely that they freeze than in our set up, a box divided in two chambers with a neutral compartment where the animals can escape. This is also true in response to a footshock, the most common fear-inducing stimulus in the laboratory. When rats are given the opportunity to escape to a save chamber, they do not freeze but quickly escape following the footshock (Blanchard et al., 2006).

Additionally, the conditions in which volatile compounds are tested determine their effective concentration in the air. Thus, if a given amount of a volatile substance is presented in a small cage covered by a lid, the concentration in the air will be higher than in an open or larger cage. This might also explain the differences found between the different studies in the behavioral response of animals to volatiles. 


\section{Exposure to pure 2-HP Elicits Avoidance and Enhanced Immobility}

To our knowledge, this is the first report analyzing the behavioral effects of 2-HP in adult female mice. Although sometimes quoted as a "known mouse pheromone" (Xu et al., 2005), studies on the behavioral and endocrine effects of 2-HP in mice are scarce, and restricted to early reports about its puberty-affecting properties (Novotny et al., 1986; Jemiolo et al., 1989). Conversely, more recent studies in rats have shown that 2-HP is elevated in the urine of stressed rats (Gutiérrez-García et al., 2006) and increases immobility in the forced swimming test (Gutiérrez-García et al., 2007), suggesting that 2-HP acts as an alarm pheromone in this species (but see also Zhang et al., 2008; Zhang and Zhang, 2011 for studies about the role of 2-HP as a sexual pheromone in rats).

The alarming properties of 2-HP have been also demonstrated in bees (Collins et al., 1989). Even if it might seem surprising that the same compound acts as a pheromone in vertebrates and insects, this is not an isolated case. For example, (Z)-7-dodecenl-yl acetate works as a pheromone in several species of moths and in elephants (Rasmussen et al., 1996). Anyway, although the avoidance observed to pure 2-HP in mice would fit its role as an alarm pheromone, it is possible that these aversive properties are due to pungent or disgusting properties of the pure substancewhich the animals are unlikely to encounter in nature-rather than to its pheromonal actions. This might represent another case of substance-unspecific aversion to an intense odor (see above).

Although 2-HP elicited avoidance only when presented pure, it decreased the distance traveled and increased immobility in a concentration-dependent way. This finding suggests that 2-HP was detected at relatively low concentrations $(0.35 \mathrm{nmol})$. However, data from habituation-dishabituation tests (Experiment 2 and Experiment S1) shows that amounts lower than $35 \mu \mathrm{mol}$ of 2 -HP did not significantly elicit chemoinvestigation. These results raise the possibility that 2 HP might have subthreshold behavioral effects. In fact, the vomeronasal organ of mice responds to 2-HP at a concentration of $10^{-11}$ in vitro (Leinders-Zufall et al., 2000). Moreover, this response is sex-specific, so that female-derived vomeronasal preparations, but not male-derived ones, responded to 2HP by increasing the intracellular concentration of inositol-3phosphate (Thompson et al., 2004), thus indicating that the aversion to the pure substance described in the present work might not be the only biological effect of this chemical signal. In summary, it is possible that the effects of 2-HP depend on the age and sex of the animals, so that it might act as a puberty regulator in pre-puber females, and as an alarm pheromone in adult females and males. The effects of 2-HP might also be strongly dependent on its concentration and on the chemical blend in which it is encountered (Novotny et al., 1986). Future studies are needed to tests these hypothesis.

Finally, the modulation of locomotor activity by IA and 2HP was similar. Our data about the responses of mice to IA are in agreement to a previous study showing that IA induced avoidance and increased immobility in rats (Wallace and Rosen, 2000). Thus, although IA is commonly used as a control odor, and our data shows that it is neutral to mice at its detection threshold, our data also suggest that it should not be used at high concentrations for that purpose, since a strong olfactory stimulation results in strong avoidance.

In summary, the similarity in the behavioral responses of mice to high concentrations of two biologically significant chemosignals and a common odorant suggest that strong odorants might induce odorant-unspecific behavioral responses of avoidance. In mice, pure TMT elicits corticosterone secretion (see Fendt et al., 2005), and a pattern of brain c-fos expression suggestive of intense stress (Janitzky et al., 2015). These findings together with our present results suggest that intense odors might be used to study stress and anxiety in addition to other unspecific stimuli such as loud noises (Burow et al., 2005; Mikheenko et al., 2010). Studies of the endocrine response to and central effects of high concentrations of control odorants such as isoamyl acetate are needed to check this hypothesis.

\section{Conditioned Contextual Responses of Mice after Repeated Exposure to TMT and IA}

The lack of consistent freezing to TMT, together with the failure to induce the contextual learning that is observed after exposure to other predator cues, i.e., cat odor (Wallace and Rosen, 2000; Blanchard et al., 2003; Muñoz-Abellán et al., 2009) have been considered by several researchers as a challenge to the kairomonal nature of this substance. Nonetheless, Endres and Fendt (2007) explored whether TMT could support learning by using different conditioning protocols. Their data showed that whereas TMT failed to induce conditioning in rats trained in a box with a single compartment [in agreement with the results obtained by Blanchard et al. (2003)], it induced conditioned avoidance in a box divided in two compartments. Our results replicate and extend these findings, since mice in our experiment expressed a conditioned avoidance of a zone paired with TMT, as well as a specific increase in immobility in the neutral zone. This latter result is striking, since TMT decreased immobility during training days. Nevertheless, as noted by (Endres and Fendt, 2007), the conditioned behavioral responses are not necessarily the same as the unconditioned ones. In addition, it is possible that our mice were able to express conditioned avoidance because they could use TMT as a discriminative stimulus due to the lack of strong innate responses toward TMT, which could interfere with conditioning in other strains. Finally, as we have stressed before, differences in the set up and protocols used might contribute to differences in the experiments outcome.

On the contrary, 2-HP failed to induce a conditioned avoidance, albeit it was, overall, significantly more avoided than TMT during the exposure days. Moreover, whereas the avoidance toward 2-HP increased the second training day, the avoidance toward TMT was slightly reduced after repeated exposures, suggesting a slight habituation. These differences are open to several interpretations.

First, it is possible that, at the high concentration used, TMT activated the trigeminal nerve (Galliot et al., 2012) producing an aversion strong enough to form an avoidance memory. In fact, although trigeminal deafferentiation does not block freezing to TMT (Ayers et al., 2013), TMT induces trigeminal activation at concentrations higher than 10\% (Hacquemand et al., 2010), at least in some experimental conditions. The exposure to IA, 
also known to stimulate the trigeminal nerve (Doty et al., 1978), mimicked the results obtained with TMT in our experiments. This suggests that a strong olfactory stimulation, maybe along with trigeminal activation, is enough to support learning, even if the odorant has, in principle, no special innate meaning for the animals. Future studies investigating memory induction with lower amounts of TMT and IA in control and anosmic animals would shed light on whether the olfactory and/or trigeminal properties of these odorants are responsible for their ability to support learning.

However, although 2-HP could also stimulate the trigeminal nerve at high concentrations (Cometto-Muñiz and Cain, 1995), and elicited aversion in mice when presented pure, it failed to induce contextual learning. Maybe 2-HP is recognized as a mouse-derived odor and, hence, it is not tagged as dangerous. Finally, whereas TMT and IA activate the main olfactory bulb only (Bepari et al., 2012), 2-HP is detected by both, the olfactory epithelium (Spehr et al., 2006) and V1R vomeronasal receptors (Boschat et al., 2002), thus activating both the main and the accessory olfactory systems (Xu et al., 2005). Given the overlapping but complementary roles of the main and accessory olfactory systems (Martínez-García et al., 2009), it is possible that higher order brain structures processing the olfactory and vomeronasal stimuli result in different unconditioned and learnt responses.

\section{Neural Basis of Biologically Significant Odor Processing}

The brain circuits processing TMT and other predator-derived cues (e.g., cat odor) might be, in fact, underlying the diversity of responses toward each type of chemosignals. Thus, cat fur odor activates the vomeronasal system (Dielenberg et al., 2001; Staples et al., 2008), including the posteroventral medial amygdala, a key center for defensive anti-predatory responses (Day et al., 2004; Pérez-Gómez et al., 2015). By contrast, TMT is not able to induce c-fos in this nucleus (Day et al., 2004; Janitzky et al., 2015; PérezGómez et al., 2015). In agreement with the activation of defensive nuclei of the brain, mice show robust risk assessment responses toward cat odor (Pérez-Gómez et al., 2015), but fail to do so when exposed to TMT (Pérez-Gómez et al., 2015, present results). In this sense, cat fur odor seems a more valuable stimulus than TMT to study antipredatory responses in rodents.

On the other hand, the bed nucleus of the stria terminalis (BNST) seems key in controlling the behavioral responses to TMT. Thus, TMT elicits robust and specific increases of Fos induction in the BNST (Janitzky et al., 2015), and temporary inactivation of this nucleus with muscimol injections abolished freezing to TMT in rats (Fendt et al., 2003). The BNST has been related to anxiety rather than fear (Davis et al., 2010). Fear is a response to an explicit threat, whereas anxiety involves uncertainty as to the expectancy of threat, and predator-derived odors are, indeed, poor predictors of the presence of a predator itself (Blanchard et al., 2003). Thus, we suggest that TMT could certainly be regarded asan anxiogenic rather than as a fear-provoking stimulus, but this hypothesis needs further investigation.

Regarding 2-HP, to our knowledge there are not studies looking at the activation of central brain structures beyond the olfactory bulbs (Xu et al., 2005). Mapping of alarm pheromones obtained from the anal glands of rats revealed Fos increases in the BNST and other nuclei involved in stress processing (Kiyokawa et al., 2005). It would be interesting that future studies directly compare the brain activation patterns elicited by both biologically significant and common of odors under the same conditions.

\section{Conclusions}

In conclusion, our results suggest that some intense odors are avoided by mice irrespective of their possible role in inter- or intra-species communication. Second, some of these intense odors, including fox-derived TMT and the odorant IA, which in principle is devoid of value for intra- or interspecies communication, induce conditioned avoidance when repeatedly presented at high concentration. By contrast, 2-HP is avoided at high concentrations but it does not induce contextual learning, maybe reflecting its role as an intraspecific chemosignal. These results support the widely use of synthetic predatorrelated compounds (like TMT) to investigate the neurobiological basis of emotional behaviors in rodents. However, since IA, a common odorant frequently used as control, elicits similar behavioral reactions to TMT, we stress the importance of using behavioral measures in combination with other physiological or neural manipulations (e.g., measurements of hormonal levels, expression of immediate early genes) to establish the ethological significance of chemosignals.

\section{Author Contributions}

EL, FM, and CA, designed research; LF and CA performed research; LF and CA analyzed data; LF, FM, and CA wrote the paper, EL, FM, and CA revised the final version and approved the manuscript.

\section{Acknowledgments}

This study was funded by the Spanish MINECO-FEDER (BFU2010-16656; BFU2013-47688-P), Junta de Comunidades de Castilla-La Mancha/FEDER (PEIC11-0045-4490), and Vicerrectorado de Investigación, Universitat Jaume I de Castelló. We would like to thank the reviewers for their fruitful suggestions, which help us improve the manuscript. The authors are indebted to Adoración Hernández-Martínez for technical assistance.

\section{Supplementary Material}

The Supplementary Material for this article can be found online at: http://journal.frontiersin.org/article/10.3389/fnins. 2015.00336 


\section{References}

Agustín-Pavón, C., Martínez-García, F., and Lanuza, E. (2014). Focal lesions within the ventral striato-pallidum abolish attraction for male chemosignals in female mice. Behav. Brain Res. 259, 292-296. doi: 10.1016/j.bbr.2013.11.020

Agustín-Pavón, C., Martínez-Ricós, J., Martínez-García, F., and Lanuza, E. (2007). Effects of dopaminergic drugs on innate pheromone-mediated reward in female mice: a new case of dopamine-independent "liking". Behav. Neurosci. 121, 920-932. doi: 10.1037/0735-7044.121.5.920

Ayers, L. W., Asok, A., Heyward, F. D., and Rosen, J. B. (2013). Freezing to the predator odor 2,4,5 dihydro 2,5 trimethylthiazoline (TMT) is disrupted by olfactory bulb removal but not trigeminal deafferentation. Behav. Brain Res. 253, 54-59. doi: 10.1016/j.bbr.2013.06.034

Bepari, A. K., Watanabe, K., Yamaguchi, M., Tamamaki, N., and Takebayashi, H. (2012). Visualization of odor-induced neuronal activity by immediate early gene expression. BMC Neurosci. 13:140. doi: 10.1186/1471-2202-13-140

Berton, F., Vogel, E., and Belzung, C. (1998). Modulation of mice anxiety in response to cat odor as a consequence of predators diet. Physiol. Behav. 65, 247-254. doi: 10.1016/S0031-9384(98)00126-7

Blanchard, D. C., Markham, C., Yang, M., Hubbard, D., Madarang, E., and Blanchard, R. J. (2003). Failure to produce conditioning with low-dose trimethylthiazoline or cat feces as unconditioned stimuli. Behav. Neurosci. 117, 360-368. doi: 10.1037/0735-7044.117.2.360

Blanchard, D. C., Yang, M., Herbert, M. A., and Blanchard, R. J. (2006). "Defensive behaviours," in Encyclopaedia of Stress, ed G. Fink (New York, NY: Academic Press), 652-656.

Boschat, C., Pélofi, C., Randin, O., Roppolo, D., Lüscher, C., Broillet, M.-C., et al. (2002). Pheromone detection mediated by a V1r vomeronasal receptor. Nat. Neurosci. 5, 1261-1262. doi: 10.1038/nn978

Brechbühl, J., Moine, F., Klaey, M., Nenniger-Tosato, M., Hurni, N., Sporkert, F., et al. (2013). Mouse alarm pheromone shares structural similarity with predator scents. Proc. Natl. Acad. Sci. U.S.A. 110, 4762-4767. doi: $10.1073 /$ pnas. 1214249110

Burow, A., Day, H. E., and Campeau, S. (2005). A detailed characterization of loud noise stress: intensity analysis of hypothalamo-pituitaryadrenocortical axis and brain activation. Brain Res. 1062, 63-73. doi: 10.1016/j.brainres.2005.09.031

Collins, A. M., Rinderer, T. E., Daly, H. V., Harbo, J. R., and Pesante, D. (1989). Alarm pheromone production by two honeybee (Apis mellifera) types. J. Chem. Ecol. 15, 1747-1756. doi: 10.1007/BF01012262

Cometto-Muñiz, J. E., and Cain, W. S. (1995). Relative sensitivity of the ocular trigeminal, nasal trigeminal and olfactory systems to airborne chemicals. Chem. Senses 20, 191-198.

Davis, M., Walker, D. L., Miles, L., and Grillon, C. (2010). Phasic vs sustained fear in rats and humans: role of the extended amygdala in fear vs anxiety. Neuropsychopharmacology 35, 105-135. doi: 10.1038/npp.2009.109

Day, H. E., Masini, C. V., and Campeau, S. (2004). The pattern of brain c-fos mRNA induced by a component of fox odor, 2,5-dihydro2,4,5-Trimethylthiazoline (TMT), in rats, suggests both systemic and processive stress characteristics. Brain Res. 1025, 139-151. doi: 10.1016/j.brainres.2004.07.079

Dewan, A., Pacifico, R., Zhan, R., Rinberg, D., and Bozza, T. (2013). Nonredundant coding of aversive odours in the main olfactory pathway. Nature 497, 486-489. doi: 10.1038/nature12114

Dielenberg, R. A., Hunt, G. E., and McGregor, I. S. (2001). "When a rat smells a cat": the distribution of Fos immunoreactivity in rat brain following exposure to a predatory odor. Neuroscience 104, 1085-1097. doi: 10.1016/S03064522(01)00150-6

Doty, R. L., Brugger, W. E., Jurs, P. C., Orndorff, M. A., Snyder, P. J., and Lowry, L. D. (1978). Intranasal trigeminal stimulation from odorous volatiles: psychometric responses from anosmic and normal humans. Physiol. Behav. 20, 175-185. doi: 10.1016/0031-9384(78)90070-7

Endres, T., and Fendt, M. (2007). Conditioned behavioral responses to a context paired with the predator odor trimethylthiazoline. Behav. Neurosci. 121, 594-601. doi: 10.1037/0735-7044.121.3.594

Endres, T., and Fendt, M. (2009). Aversion- vs fear-inducing properties of 2,4,5trimethyl-3-thiazoline, a component of fox odor, in comparison with those of butyric acid. J. Exp. Biol. 212, 2324-2327. doi: 10.1242/jeb.028498
Fendt, M., and Endres, T. (2008). 2,3,5-Trimethyl-3-thiazoline (TMT), a component of fox odor - just repugnant or really fear-inducing? Neurosci. Biobehav. Rev. 32, 1259-1266. doi: 10.1016/j.neubiorev.2008.05.010

Fendt, M., Endres, T., and Apfelbach, R. (2003). Temporary inactivation of the bed nucleus of the stria terminalis but not of the amygdala blocks freezing induced by trimethylthiazoline, a component of fox feces. J. Neurosci. 23, 23-28. Available online at: http://www.jneurosci.org/content/23/1/23.long

Fendt, M., Endres, T., Lowry, C. A., Apfelbach, R., and McGregor, I. S. (2005). TMT-induced autonomic and behavioral changes and the neural basis of its processing. Neurosci. Biobehav. Rev. 29, 1145-1156. doi: 10.1016/j.neubiorev.2005.04.018

Ferrero, D. M., Lemon, J. K., Fluegge, D., Pashkovski, S. L., Korzan, W. J., Datta, S. R., et al. (2011). Detection and avoidance of a carnivore odor by prey. Proc. Natl. Acad. Sci. U.S.A. 108, 11235-11240. doi: 10.1073/pnas.1103317108

Fortes-Marco, L., Lanuza, E., and Martinez-Garcia, F. (2013). Of pheromones and kairomones: what receptors mediate innate emotional responses? Anat. Rec. 296, 1346-1363. doi: 10.1002/ar.22745

Galliot, E., Laurent, L., Hacquemand, R., Pourié, G., and Millot, J. L. (2012). Fearlike behavioral responses in mice in different odorant environments: trigeminal versus olfactory mediation under low doses. Behav. Processes 90, 161-166. doi: 10.1016/j.beproc.2012.01.002

Gutiérrez-García, A. G., Contreras, C. M., Mendoza-López, M. R., Cruz-Sánchez, J. S., García-Barradas, O., and Rodríguez-Landa, J. (2006). A single session of emotional stress produces anxiety in Wistar rats. Behav. Brain Res. 167, 30-35. doi: 10.1016/j.bbr.2005.08.011

Gutiérrez-García, A. G., Contreras, C. M., Mendoza-López, M. R., GarcíaBarradas, O., and Cruz-Sánchez, J. S. (2007). Urine from stressed rats increases immobility in receptor rats forced to swim: role of 2-heptanone. Physiol. Behav. 91, 166-172. doi: 10.1016/j.physbeh.2007.02.006

Hacquemand, R., Choffat, N., Jacquot, L., and Brand, G. (2013). Comparison between low doses of TMT and cat odor exposure in anxiety- and fear-related behaviors in mice. Behav. Brain Res. 238, 227-231. doi: 10.1016/j.bbr.2012.10.014

Hacquemand, R., Jacquot, L., and Brand, G. (2010). Comparative fear-related behaviors to predator odors (TMT and Natural Fox Feces) before and after intranasal $\mathrm{ZnSO}(4)$ treatment in mice. Front. Behav. Neurosci. 4:188. doi: 10.3389/fnbeh.2010.00188

Hebb, A. L., Zacharko, R. M., Gauthier, M., Trudel, F., Laforest, S., and Drolet, G. (2004). Brief exposure to predator odor and resultant anxiety enhances mesocorticolimbic activity and enkephalin expression in CD-1 mice. Eur. J. Neurosci. 20, 2415-2429. doi: 10.1111/j.1460-9568.2004.03704.x

Janitzky, K., D’Hanis, W., Kröber, A., and Schwegler, H. (2015). TMT predator odor activated neural circuit in C57BL/6J mice indicates TMT-stress as a suitable model for uncontrollable intense stress. Brain Res. 1599, 1-8. doi: 10.1016/j.brainres.2014.12.030

Jemiolo, B., Alberts, J., Sochinski-Wiggins, S., Harvey, S., and Novotny, M. (1985). Behavioural and endocrine responses of female mice to synthetic analogues of volatile compounds in male urine. Anim. Behav. 33, 1114-1118. doi: 10.1016/S0003-3472(85)80170-6

Jemiolo, B., Andreolini, F., Xie, T. M., Wiesler, D., and Novotny, M. (1989). Puberty-affecting synthetic analogs of urinary chemosignals in the house mouse, Mus domesticus. Physiol. Behav. 46, 293-298. doi: 10.1016/00319384(89)90270-9

Kiyokawa, Y., Kikusui, T., Takeuchi, Y., and Mori, Y. (2005). Mapping the neural circuit activated by alarm pheromone perception by c-Fos immunohistochemistry. Brain Res. 1043, 145-154. doi: 10.1016/j.brainres.2005.02.061

Leinders-Zufall, T., Lane, A. P., Puche, A. C., Ma, W., Novotny, M. V., Shipley, M. T., et al. (2000). Ultrasensitive pheromone detection by mammalian vomeronasal neurons. Nature 405, 792-796. doi: 10.1038/35015572

Martínez-García, F., Martínez-Ricós, J., Agustín-Pavón, C., Martínez-Hernández, J., Novejarque, A., and Lanuza, E. (2009). Refining the dual olfactory hypothesis: pheromone reward and odour experience. Behav. Brain Res. 200, 277-286. doi: 10.1016/j.bbr.2008.10.002

Martínez-Ricós, J., Agustín-Pavón, C., Lanuza, E., and Martínez-García, F. (2007). Intraspecific communication through chemical signals in female mice: reinforcing properties of involatile male sexual pheromones. Chem. Senses 32, 139-148. doi: 10.1093/chemse/bj1039 
McClintock, M. K. (2002). "Pheromones, odors and vasanas: the neuroendocrinology of social chemosignals in humans and animals," in Hormones, Brain and Behavior, eds D. W. Pfaff, A. P. Arnold, A. M. Etgen, S. E. Fahrbach, and R. T. Rubin (San Diego, CA: Academic Press), 139-214.

Mikheenko, Y., Man, M.-S., Braesicke, K., Johns, M. E., Hill, G., AgustínPavón, C., et al. (2010). Autonomic, behavioral, and neural analyses of mild conditioned negative affect in marmosets. Behav. Neurosci. 124, 192-203. doi: $10.1037 / \mathrm{a} 0018868$

Morrow, B. A., Elsworth, J. D., and Roth, R. H. (2002). Fear-like biochemical and behavioral responses in rats to the predator odor, TMT, are dependent on the exposure environment. Synapse 46, 11-18. doi: 10.1002/syn.10109

Muñoz-Abellán, C., Andero, R., Nadal, R., and Armario, A. (2008). Marked dissociation between hypothalamic-pituitary-adrenal activation and longterm behavioral effects in rats exposed to immobilization or cat odor. Psychoneuroendocrinology 33, 1139-1150. doi: 10.1016/j.psyneuen.2008.06.002

Muñoz-Abellán, C., Daviu, N., Rabasa, C., Nadal, R., and Armario, A. (2009). Cat odor causes long-lasting contextual fear conditioning and increased pituitaryadrenal activation, without modifying anxiety. Horm. Behav. 56, 465-471. doi: 10.1016/j.yhbeh.2009.08.002

Novotny, M., Jemiolo, B., Harvey, S., Wiesler, D., and Marchlewska-Koj, A. (1986). Adrenal-mediated endogenous metabolites inhibit puberty in female mice. Science 231, 722-725. doi: 10.1126/science.3945805

Pérez-Gómez, A., Bleymehl, K., Stein, B., Pyrski, M., Birnbaumer, L., Munger, S. D., et al. (2015). Innate predator odor aversion driven by parallel olfactory subsystems that converge in the ventromedial hypothalamus. Curr. Biol. 25, 1340-1346. doi: 10.1016/j.cub.2015.03.026

Rasmussen, L. E. L., Lee, T. D., Roelofs, W. L., Zhang, A., and Daves, G. D. (1996). Insect pheromone in elephants. Nature 379, 684. doi: 10.1038/379684a0

Root, C. M., Denny, C. A., Hen, R., and Axel, R. (2014). The participation of cortical amygdala in innate, odor-driven behavior. Nature 515, 269-273. doi: 10.1038 /nature 13897

Rosen, J. B., West, E. A., and Donley, M. P. (2006). Not all rat strains are equal: differential unconditioned fear responses to the synthetic fox odor 2,4,5trimethylthiazoline in three outbred rat strains. Behav. Neurosci. 120, 290-297. doi: 10.1037/0735-7044.120.2.290

Spehr, M., Kelliher, K. R., Li, X.-H., Boehm, T., Leinders-Zufall, T., and Zufall, F. (2006). Essential role of the main olfactory system in social recognition of major histocompatibility complex peptide ligands. J. Neurosci. 26, 1961-1970. doi: 10.1523/JNEUROSCI.4939-05.2006
Staples, L. G., McGregor, I. S., Apfelbach, R., and Hunt, G. E. (2008). Cat odor, but not trimethylthiazoline (fox odor), activates accessory olfactory and defense-related brain regions in rats. Neuroscience 151, 937-947. doi: 10.1016/j.neuroscience.2007.11.039

Thompson, R. N., Robertson, B. K., Napier, A., and Wekesa, K. S. (2004). Sexspecific responses to urinary chemicals by the mouse vomeronasal organ. Chem. Senses 29, 749-754. doi: 10.1093/chemse/bjh076

Vernet-Maury, E. (1980). Trimethyo-thiazoline in fox feces: a natural alarming substance for the rat. Olfaction Tast. 7, 407.

Wallace, K. J., and Rosen, J. B. (2000). Predator odor as an unconditioned fear stimulus in rats: elicitation of freezing by trimethylthiazoline, a component of fox feces. Behav. Neurosci. 114, 912-922. doi: 10.1037/0735-7044. 114.5.912

Wernecke, K. E. A., Vincenz, S., Storsberg, S., D’Hanis, W., Goldschmidt, J., and Fendt, M. (2015). Fox urine exposure induces avoidance behavior in rats and activates the amygdalar olfactory cortex. Behav. Brain Res. 279, 76-81. doi: 10.1016/j.bbr.2014.11.020

Xu, F., Schaefer, M., Kida, I., Schafer, J., Liu, N., Rothman, D. L., et al. (2005). Simultaneous activation of mouse main and accessory olfactory bulbs by odors or pheromones. J. Comp. Neurol. 489, 491-500. doi: 10.1002/cne. 20652

Zhang, J. X., Sun, L., Zhang, J. H., and Feng, Z. Y. (2008). Sex- and gonad-affecting scent compounds and 3 male pheromones in the rat. Chem. Senses 33, 611-621. doi: 10.1093/chemse/bjn028

Zhang, Y. H., and Zhang, J. X. (2011). Urine-derived key volatiles may signal genetic relatedness in male rats. Chem. Senses 36, 125-135. doi: $10.1093 /$ chemse/bjq103

Conflict of Interest Statement: The authors declare that the research was conducted in the absence of any commercial or financial relationships that could be construed as a potential conflict of interest.

Copyright (c) 2015 Fortes-Marco, Lanuza, Martínez-García and Agustín-Pavón. This is an open-access article distributed under the terms of the Creative Commons Attribution License (CC BY). The use, distribution or reproduction in other forums is permitted, provided the original author(s) or licensor are credited and that the original publication in this journal is cited, in accordance with accepted academic practice. No use, distribution or reproduction is permitted which does not comply with these terms. 